\title{
Optic Nerve Movement May Need to be Considered When Treating with Stereotactic Radiosurgery
}

\author{
Kathryn Clarke ${ }^{1}$, Gerald B Fogarty ${ }^{1,2,3^{*}}$, Serigne Lo $^{2,3}$, Michael Izard ${ }^{1}$ and Angela Hong ${ }^{1,2,3}$ \\ ${ }^{1}$ Genesis Cancer Care, Department of Radiation Oncology, The Mater Hospital, Rocklands Road, Crows Nest NSW 2065, Australia \\ ${ }^{2}$ Melanoma Institute Australia, Sydney, Australia \\ ${ }^{3}$ Sydney Medical School, The University of Sydney, Sydney, Australia
}

"Corresponding author: Gerald B Fogarty, Genesis Cancer Care, Department of Radiation Oncology, The Mater Hospital, Rocklands Road, Crows Nest NSW 2065, Australia, Tel: 612 94588050, E-mail: gerald.fogarty@cancer.com.au

Received: November 14, 2016; Accepted: December 12, 2016; Published: January 01, 2017

Copyright: (c) 2017 Clarke $\mathrm{K}$, et al. This is an open-access article distributed under the terms of the Creative Commons Attribution License, which permits unrestricted use, distribution, and reproduction in any medium, provided the original author and source are credited.

\begin{abstract}
Introduction: Fractionated stereotactic radiosurgery (fSRS) is an effective definitive treatment for optic nerve (ON) tumors. It is controversial whether treatment to the ON requires attention to the position of the eye as some consider the movement to be negligible. We investigated in four patients to establish whether there is clinically significant displacement of the $\mathrm{ON}$ as the globe moves and the effect this may have on target coverage in fSRS treatments.

Method: Four patients receiving radiotherapy treatment to the skull and requiring cone beam computed tomography (CBCT) matching were asked to look in different directions as routine CBCT were obtained. An fSRS plan was created, treating the $\mathrm{ON}$ with the patient looking straight ahead. The displacement of the $\mathrm{ON}$ from straight was measured, with the patient looking fully left or right, the DVH of the optic nerve in the three locations was compared.
\end{abstract}

Results: The data collected showed that there could be a significant difference in optic nerve position as the patient's gaze changed. The DVH showed that the PTV coverage was affected as the patient moved their gaze either left or right by up to $9 \%(95 \% \mathrm{Cl}$ is from $-15 \%$ to $-3 \%, \mathrm{p}=0.012)$.

Conclusion: As a result of our study we recommend cooperative patients receive some training to ensure that optic nerve positioning is maintained throughout simulation and treatment.

Keywords: Optic nerve; Stereotactic; Radiosurgery; Radiation therapy

\section{Introduction}

Fractionated stereotactic radiosurgery (fSRS) is an effective definitive treatment for optic nerve (ON) tumors such as ON glioma and ON sheath meningioma's. It is crucial to the success of the treatment that the patient is sufficiently stabilized in simulation and then throughout treatment. It is controversial whether treatment to the $\mathrm{ON}$ requires attention to the position of the eye, as some consider the movement to be negligible. We wondered whether patients can unintentionally affect coverage of their optic nerve by altering their gaze during treatment.

We investigated in four patients to establish whether there is clinically significant displacement of the $\mathrm{ON}$ as the globe moves, and the effect this may have on target coverage in stereotactic radiosurgery treatments of the ON.

\section{Method}

Four randomly selected patients, with full movement of their globes, receiving treatment to the skull at the Mater in 2015 were asked to keep their gaze in the neutral position or straight as their simulation computed tomography (CT) was obtained. When scheduled cone beam computed tomography (CBCT) scans were conducted, the patients were asked to look fully to their left for one scan, and fully to the right for the next. All data sets were imported into Varian Eclipse for planning. Each patient then had three CT data sets. The three CT data sets for each patient were fused so that the position of the ONs at the three eye positions could be compared. The position of the $\mathrm{ON}$ as the patient's gaze was neutral or straight was used to create a clinical target volume (CTV) as per ICRU 52/60 [1]. As this was a retrospective study using patients not necessarily for treatment for optic glioma or ON sheath meningioma, a gross tumor volume (GTV) could not be volumed. A fSRS treatment with a prescription of $50.4 \mathrm{~Gy}$ in 28 fractions was planned with Eclipse's Rapid Arc using two full arcs. All plans were considered clinically acceptable according to guidelines outlined by the British Neuro-Oncology Society [2]. Both ONs of all four patients as they looked fully to the right or fully to the left were also contoured and volumes calculated. Measurement points were created at the proximal, middle and distal points along the length of each optic nerve (Figure 1) in each of the positions - neutral, globe looking fully to left and fully to right, for both optic nerves of all four patients, a total of four by two by nine, that is, 72 data points for significance analysis. Using the straight $\mathrm{ON}$ as a baseline, the displacement of the points from this to those of the deviated nerves were measured and recorded. 
Citation: Clarke K, Fogarty GB, Lo S, Izard M, Hong A (2017) Optic Nerve Movement May Need to be Considered When Treating with Stereotactic Radiosurgery. J Nucl Med Radiat Ther 8: 321. doi:10.4172/2155-9619.1000321

Page 2 of 3

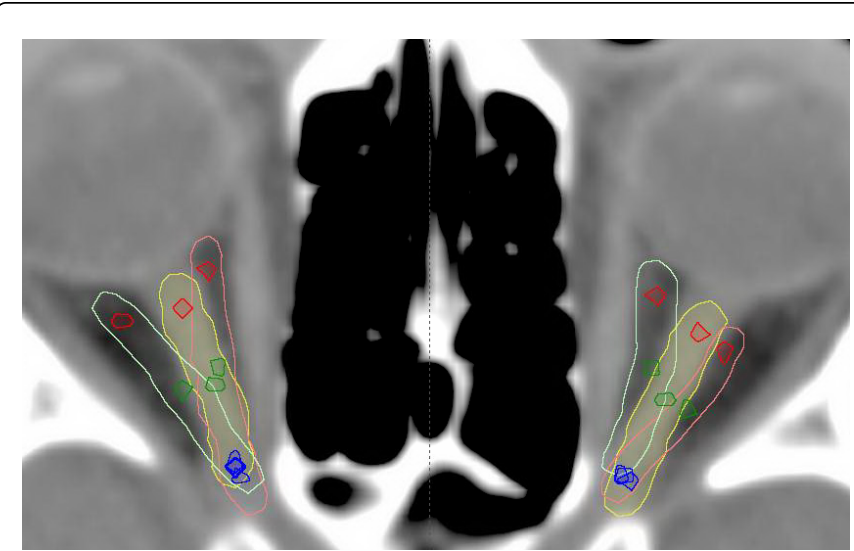

Figure 1: Optic nerve structures and measurement points. The yellow optic nerves are as the patient looks straight, pink optic nerves are as the patients looks right, and light green is the optic nerves are the patients looks left. The red objects are distal measurement points, green are mid-length measurement points, and blue are proximal measurement points.

According to ICRU 50/62 protocol, the minimum acceptable dose coverage of the PTV is $95 \%$. A dose volume histogram (DVH) was used to compare the percentage of the ON covered by the $95 \%$ isodose volume of prescribed dose with eye in different positions (Figure 2). A mixed linear model was used to estimate the average percentage of the 95\% isodose line that the ON received when the gaze was left, straight or right, with straight assumed to be the reference. The linear mixed model allows adjustment for the correlation between the various volumes coverage taken from the same individual. No other covariates were included in the analysis to avoid over-fitting the model due to the limited sample size [3-6].

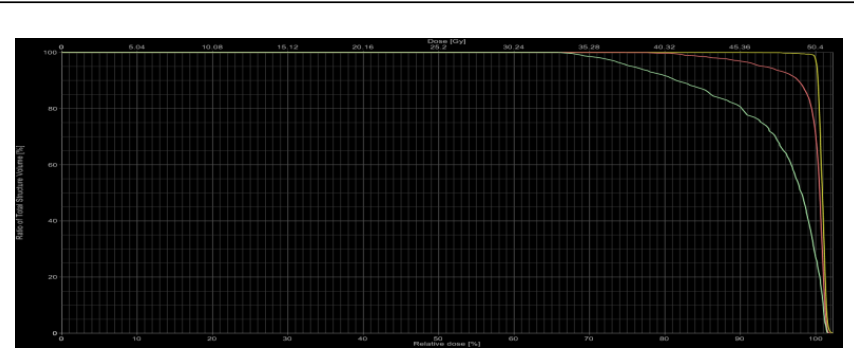

Figure 2: Dose volume histogram displaying the right optic nerves with the patient looking right (pink), left (green) and straight (yellow).

\section{Results}

Table 1 shows the displacement of the right optic nerves by comparing points along its length using the $\mathrm{ON}$ when the patient is looking straight as a baseline. The largest displacement was seen in patient $\mathrm{B}$ when their gaze was to the left. At the distal measurement point (the point closest to the globe) there was a lateral displacement of $6 \mathrm{~mm}$. For most patients, there was small or no displacement of the right $\mathrm{ON}$ at the proximal measurement point. Table 2 displays the displacement of the left optic nerves. Again, patient B had the largest displacement of $6 \mathrm{~mm}$ as their gaze was to the left. Patient A also had a $6 \mathrm{~mm}$ displacement of the left $\mathrm{ON}$ when looking to the right.

\begin{tabular}{|c|c|c|c|c|c|c|c|}
\hline Patient & $\begin{array}{l}\text { Distal } \\
\text { looking left } \\
(\mathrm{mm})\end{array}$ & $\begin{array}{l}\text { Mid } \\
\text { looking left } \\
(\mathrm{mm})\end{array}$ & $\begin{array}{l}\text { Proximal looking } \\
\text { left }(\mathrm{mm})\end{array}$ & $\begin{array}{l}\text { Baseline=Optic } \quad \text { Nerve } \\
\text { looking Straight }\end{array}$ & $\begin{array}{l}\text { Distal looking } \\
\text { right }(\mathrm{mm})\end{array}$ & $\begin{array}{l}\text { Mid } \\
\text { looking right } \\
(\mathrm{mm})\end{array}$ & $\begin{array}{l}\text { Proximal looking } \\
\text { right }(\mathrm{mm})\end{array}$ \\
\hline A & -2 & -1 & -1 & $0 \mathrm{~mm}$ displacement & 4 & 2 & 0.5 \\
\hline B & -6 & -3 & 0 & $0 \mathrm{~mm}$ displacement & 3 & 1 & 0 \\
\hline C & -2 & -1 & 0 & $0 \mathrm{~mm}$ displacement & 4 & 1 & 0 \\
\hline D & -1 & -1 & 0 & $0 \mathrm{~mm}$ displacement & 1 & 1 & 1 \\
\hline
\end{tabular}

Table 1: Displacement of right optic nerves (in mm).

\begin{tabular}{|c|c|c|c|c|c|c|c|c|}
\hline Patient & $\begin{array}{l}\text { Distal } \\
\text { looking left } \\
(\mathrm{mm})\end{array}$ & $\begin{array}{l}\text { Mid looking } \\
\text { left }(\mathrm{mm})\end{array}$ & $\begin{array}{l}\text { Proximal looking } \\
\text { left }(\mathrm{mm})\end{array}$ & $\begin{array}{l}\text { Baseline=Optic } \quad \text { Nerve } \\
\text { looking Straight }\end{array}$ & $\begin{array}{l}\text { Distal looking } \\
\text { right }(\mathrm{mm})\end{array}$ & $\begin{array}{l}\text { Mid looking } \\
\text { right }(\mathrm{mm})\end{array}$ & $\begin{array}{l}\text { Proximal } \\
\text { right }(\mathrm{mm})\end{array}$ & looking \\
\hline A & -1.5 & -1 & 0 & $0 \mathrm{~mm}$ displacement & 6 & 1 & 0 & \\
\hline B & -6 & -3 & -1 & $0 \mathrm{~mm}$ displacement & 3 & 3 & 0 & \\
\hline C & -3 & -1 & 0 & $0 \mathrm{~mm}$ displacement & 3 & 1 & 0 & \\
\hline D & 0 & -1 & -2 & $0 \mathrm{~mm}$ displacement & 2 & 2 & 1 & \\
\hline
\end{tabular}

Table 2: Displacement of left optic nerves (in $\mathrm{mm}$ ).

The DVH was used to calculate the percentage of the optic nerve receiving $95 \%$ isodose coverage as the patient looked straight, left and right. Table 3 shows the percentage of the right optic nerves receiving coverage by the $95 \%$ isodose volume. As per protocol, $100 \%$ of the 
Citation: Clarke K, Fogarty GB, Lo S, Izard M, Hong A (2017) Optic Nerve Movement May Need to be Considered When Treating with

Page 3 of 3

right optic nerves were covered by $95 \%$ of the prescribed dose on the CT data sets with the patients looking straight. As little as $68.1 \%$ of patient B's right optic nerve received $95 \%$ coverage. Table 4 shows the coverage of the left optic nerves.

Compared to the straight ON which received $100 \%$ coverage for all plans, there is an average coverage reduction of $9 \%(95 \% \mathrm{CI}$ is from $-15 \%$ to $-3 \%, \mathrm{p}=0.012$ ) when the $\mathrm{ON}$ is looking left while when the ON is looking right the coverage is reduced by $7 \%(95 \% \mathrm{CI}$ is from $-14 \%$ to $-1.5 \%, \mathrm{p}=0.028$ ).

\begin{tabular}{|c|c|c|c|}
\hline Patient & Looking Left & Looking Straight & Looking Right \\
\hline A & 92.7 & 100 & 92.7 \\
\hline B & 68.1 & 100 & 93.5 \\
\hline C & 99 & 100 & 82.3 \\
\hline D & 100 & 100 & 100 \\
\hline
\end{tabular}

Table 3: Coverage of the right optic nerves (in percent) by $95 \%$ of the prescribed dose.

\begin{tabular}{|c|c|c|c|}
\hline Patient & Looking Left & Looking Straight & Looking Right \\
\hline A & 99.7 & 100 & 92.7 \\
\hline B & 32.1 & 100 & 85.8 \\
\hline C & 36.4 & 100 & 92.7 \\
\hline D & 100 & 100 & 99 \\
\hline
\end{tabular}

Table 4: Coverage of the left optic nerves (in percent) by $95 \%$ of the prescribed dose.

\section{Conclusion}

There is controversy around whether extra effort can or should be made to ensure stability of globe and ON stability as some consider the movement to be negligible. The results obtained from our study show that there can be significant displacement of the optic nerve as the patient changes their gaze. This may lead to an under dosage. The extent of the displacement is patient dependent.

In our opinion patients do need to be asked to look ahead in neutral gaze during beam-on time. There may be additional benefit in asking the patient to keep their eyes closed, as they are then not distracted by the movement of the treatment machine or items in the treatment bunker.

\section{Ethics and Consent}

This project is a sub study of the "Whole brain radiotherapy following local treatment of intracranial metastases of melanoma - A randomized phase III trial (WBRT Study)", Protocol No. X13-0329 was approved on $9^{\text {th }}$ October 2013 by HREC/13/RPAH/465.

\section{Availability of Data and Materials}

Data collected came directly from Eclipse version 11.0.3. Deidentified raw data in the form of dose volume histograms can be sourced for distribution.

\section{Authors' Contributions}

$\mathrm{KC}$ performed scans on patients to obtain imaging for planning and analysis, created treatment plans for analysis and drafted manuscript. GBF conceived study and participated in its design and helped to draft the manuscript. SL analyzed and converted raw data into table and graphical form. GBF, MAI and AH suggested patients for inclusion and provided feedback and editing of manuscript. All authors approved the final manuscript.

\section{Acknowledgements}

We would like to acknowledge Genesis Cancer Care and the staff at Mater Radiation Oncology - Crow's Nest department for assisting in data collection. We also acknowledge Cancer Australia in supporting the Australia \& New Zealand Melanoma Trials Group (ANZMTG).

\section{References}

1. ICRU (1993) Prescribing, Recording, and Reporting Photon Beam Therapy (Report 50), Bethesda, MD, USA.

2. (2011) Guidelines on the diagnosis and management of Optic Pathway Glioma (OPG), British Neuro-Oncology Society/National Cancer Action Team.

3. Fedele D, Ponti E, Tolu B, Ingrosso G, Hamoud E, et al. (2012) Radiation Therapy in Optic Gliomas: A Case Report and a Review of Literature. J Nucl Med Radiat Ther 3: 134.

4. Kortmann R, Timmermann B, Tayor R, Scarzello G, Plasswilm L, et al. (2003) Current and Future Strategies in Radiotherapy of Childhood LowGrade Glioma of the Brain. Part 1: Treatment Modalities of Radiation Therapy. Strahlenther Onkol 179: 509-520.

5. Boulos PT, Dumont AS, Mandell JW, Jane JA (2001) Meningiomas of the orbit: contemporary considerations. Neurosurg Focus 10: 1-10.

6. Jaywant S, Osei E, Ladak S (2003) Stereotactic Radiotherapy in the Treatment of Ocular Melanoma: A non-invasive eye fixation aid and tracking system. J Appl Clin Med Phys 4: 156-161. 